\title{
Arbeitswochen für Ortsplanung in Zusammenarbeit mit einem Planungsbüro
}

Je eine 6. Klasse (12. Schuljahr) des Freien Gymnasiums Zürich führte 1969 in Baltenswil (Gemeinde Bassersdorf), 1970 in Einsiedeln und 1974 in Sempach eine Arbeitswoche durch, in der die Schülerinnen und Schüler Einblick in die Probleme einer Ortsplanung erhielten. Der Stoff wurde nicht in Vorträgen geboten oder im Unterricht erarbeitet und nachher von den Schülern reproduziert, sondern die Schüler konnten an einem Beispiel selbständig arbeiten. Sie waren dabei direkt mit den Problemen konfrontiert und und mussten selber zu Lösungsvorschlägen kommen. Die Zusammenarbeit mit dem Planungsbüro Steiger/Huber, das in den erwähnten Gemeinden den Auftrag erhalten hatte, eine Ortsplanung durchzuführen oder, wie in Sempach, eine Ortsplanung zu revidieren, ermöglichte es, dass die Resultate im weiteren Verlauf der Planung verwendet werden konnten. Die Forderung nach einem projektbezogenen Unterricht war so erfüllt. Schöpferische Fähigkeiten der Schüler kamen zum Zug. Sie arbeiteten oft bis spät in die Nacht, viele zehn und mehr Stunden im Tag, sodass die Arbeitswochen zu einem vollen Erfolg wurden.

Schon in kurzer Zeit werden die Gymnasiasten volljährig und haben als Stimmbürgerinnen und Stimmbürger in ihrer Wohngemeinde zu ähnlichen Fragen Stellung zu nehmen. Diese Arbeitswochen dienen der staatsbürgerlichen Erziehung, indem die Schüler selber erleben, wie ein ausgewogener Richtplan entsteht, der dem Einzelnen zwar Einschränkungen auferlegt, ihm aber auch viel Nutzen bringt, weil Ordnung im Zusammenleben der Menschen notwendig und vorteilhaft ist.

Sehr wertvoll war, dass die Arbeitswochen gemeinsam mit Dr. Gerhard Frick, der in den betreffenden Klassen Deutsch und Geschichte unterrichtete, durchgeführt werden konnten.

Er befasste sich vor allem auch mit der Formulierung der Texte.

Die Klassen wurden in Arbeitsgruppen aufgeteilt, wobei die Schüler die Gruppe wählen konnten. In Sempach waren es zum Beispiel sechs:

1. Umfrage: Ein von Spezialisten für Meinungsumfragen ausgearbeiteter umfangreicher Fragebogen ermöglichte eine umfassende Analyse der Wohnverhältnisse, Einkaufsgewohnheiten, Arbeitsplätze, Verkehrsverhältnisse, Vereins- zugehörigkeit, Integrationsaussichten für Neuzuzüger, usw. Obschon wir dieser Arbeitsgruppe sechs Schüler zuteilen konnten, gelang es nicht, eine genügend grosse $Z$ ahl von Interviews zu bekommen, um eine einwandfreie statistische Auswertung vorzunehmen. Wir mussten uns mit 71 Umfragen begnügen.

2. Städtli: Die Häuser im alten Stadtteil wurden bewertet nach ihrer Erhaltungswürdigkeit. Die Ergebnisse sind in einem Plan eingetragen, der auch eine andere Verkehrsführung vorschlägt um die schöne Hauptstrasse zu entlasten und als Einkaufsstrasse aufzuwerten. Um den Stellenwert einzelner, heute weniger gut unterhaltener Gebäude zu zeigen, wurde von einer Strassenseite eine Ansichtszeichnung in Parallelperspektive erstellt. Die störenden Renovationen, Umbauten und Neubauten sind sofort zu erkennen und zeigen die Folgen allfälliger neuer Eingriffe. An einem Nachmittag stand der kantonale Denkmalpfleger für alle aufgetretenen Fragen zur Verfügung dieser Arbeitsgruppe.

3. Neue Quartiere: Die Arbeits gruppe prüfte die Wirksamkeit der alten Bauordnung an den neuen Quartieren. Sie beurteilte die bestehenden Ueberbauungen nach der Qualität der Siedlung und machte Verbesserungsvorschläge sowie Vorschläge für künftige Ueberbauungen. 4. Handel, Gewerbe und öffentliche Einrichtungen: Untersucht wurden Gewerbebetriebe, Detailhandel, Läden, Preisvergleiche mit Sursee, Auswirkungen eines allfällig zu erstellenden Einkaufszentrums.

5. Landschaftsplan: Schutzgebiete, Wegnetz, geplante Autobahnraststätte.

6. Lärmschutz:Die Gruppe führte zuerst Lärm messungen mit mehreren Geräten an bestehenden Autobahnen durch. Gemessen wurde im Freien und auch in Gebäuden gleichzeitig in verschieden hohen Stockwerken. Dann erarbeiteten sie für die geplante Autobahnstrecke der N2 auf dem Gemeindegebiet von Sempach die nötigen Schutzmassnahmen, wie Aenderungen im Zonenplan, Anordnung gewerblicher Bauten längs der Autobahn, Lärmschutzwälle, Autobahnanschluss usw. Sie zeichnete die entsprechenden Det ailprofile.

Dr. S. Wyder, Freies Gymnasium Zürich, Arbenzstrasse 19, 8008 Zürich 
Die Formulierung der Probleme erfolgte durch das Planungsbüro Planpartner AG, M. Steiger und L. Huber. An drei Sitzungen vor der Arbeitswoche erarbeiteten Planer und Lehrer gemeinsam die Aufteilung der Probleme auf die einzelnen Arbeitsgruppen und das methodische Vorgehen. Es ist unbedingt nötig, dass der Lehrer die Gemeinde und die weitere Umgebung bereits vor diesen Besprechungen gut kennt und die Hauptprobleme erfasst hat. Er muss auch die wichtigste Literatur kennen. Hier liegt die Bedeutung der Fortbildungskurse des Vereins Schweiz. Geographielehrer. Ohne sie wäre der Lehrer kaum fähig aktiv mitzuarbeiten, und er wäre kein Gesprächspartner, weder bei den um Rat suchenden Schülern noch bei den Planern. Die Schüler sollten in jeder Arbeitsgruppe zu eigenen Lösungen und Formulierungen kommen. Deshalb gab ihnen der begleitende Planer keine Instruktionen. Er stand aber jeden Tag zu einer bestimmten Zeit für alle Fragen zur Verfügung. Wenn eine Gruppe nicht mehr weiter kam, schickten wir sie in eine der umliegenden Gemeinden, in der das betreffende Problem klarer sichtbar war oder wo bereits eine Lösung gefunden wurde. Unsere Planer hatten zudem eine Handbibliothek aufgebaut mit den wichtigsten Lehrbüchern und zahlreichen Berichten von andern Ortsplanungen. Da nur eine Woche zur Verfügung stand, musste äusserst rationell gearbeitet werden. Besonders wichtig war, dass jede Arbeitsgruppe sofort beginnen konnte. Bewährt hat sich folgendes Vorgehen:Nach Bezug der Unterkunft wurde ein kurzer Rundgang durch die Gemeinde unternommen. Den Schülern wurden nur ganz wenige konkrete Angaben gemacht, wie z. B. Baujahr, Ausnützungsziffer in den verschiedenen Quartieren, aber keine Probleme genannt. In Sempach gingen die Schüler allein auf den Rundgang. Nachher kam die ganze Klasse zusammen und die Schüler formulierten selber die wichtigsten Probleme der Gemeinde. Schwieriger war es dann, im Laufe der Woche Lösungsvorschläge zu finden. Die Arbeits gruppen konnten schon am ersten Morgen mit ihrer eigenen Arbeit beginnen. Am gemeinsamen Mittagessen kamen bereits die ersten gezielten Fragen. Als Arbeitsraum eigneten sich grosse Räume wie zwei nebeneinander liegende Schulzimmer oder der grosse Saal eines Wirtshauses. Jede Gruppe hatte ihren bestimmten Platz, wo sie die Pläne ausbreiten konnte, und wo sie von den andern
Gruppen gut erreicht werden konnte. Das erleichterte die Koordination. Am Abend, etwa eine Stunde vor dem Nachtessen versammelten sich alle Gruppen im Saal. Jede berichtete über ihre Ergebnisse, und was sie weiter unternehmen wolle. Dadurch erhielten die Schüler Einblick in die ganze Planung, nicht nur in die Arbeit ihrer Gruppe. Der Lehrer bekam jeden Tag eine klare Uebersicht und konnte zurückgebliebenen Gruppen helfen. Einmal hatten wir eine eigentliche Koordinationsgruppe, die ihre Kameraden mit Hilfe eines einfachen Netzplanes beraten konnte. Da zahlreiche Probleme mehrere Arbeitsgruppen beschäftigen, können so Doppelspurigkeiten vermieden werden. Die Ergebnisse nützen niemandem etwas, wenn sie nicht klar formuliert und auf Plänen festgehalten werden können. Hier brauchten die Schüler etwas Hilfe. Alle Hilfsmittel wie eine, - besser zwei, - Schreibmaschinen pro Arbeitsgruppe, Vervielfältigungsmöglichkeit, Zeichenstifte in den vom Planungsbüro angewandten Farbtönen, Rasterpapiere, genügend Pläne verschiedener Massstäbe wurden im Voraus bereitgestellt, eine Lichtpausanstalt und ein Photograph getestet, ob sie in der Lage sind, rasch zu arbeiten. Am letzten Tag musste der Bericht fertig vorliegen, sodass er nachher vervielfältigt werden konnte. Die Schüler sollten weder vor noch nach der Arbeitswoche mit Planungsaufgaben belastet sein. Wir haben zu wenig Geographiestunden um sie dafür zu verwenden und mit Hausaufgaben, wie zum Beispiel das nachträgliche Abfassen von Berichten, sollten die Schüler nicht belastet werden. Sie benötigen nach der Arbeitswoche wieder Zeit für die andern Fächer. Das sind wir unsern Kollegen schuldig.

In allen drei Arbeitswochen war es möglich am Freitag Nachmittag die Ergebnisse mit Vertretern des Gemeinderates und mit dem $\mathrm{Ge}$ meindeschreiber zu diskutieren. Das ist sehr wichtig, weil dadurch die Arbeit ihren Sinn bekommt. Die Schüler müssen sich überlegen, ob ihre Vorschläge realisierbar sind. Utopische Vorschläge, wie sie Jugendliche oft äussern, lagen deshalb kaum vor.

Schüler und Lehrer vermögen grossen Gewinn aus einer solchen Arbeitswoche zu ziehen. Ist es aber nicht vermessn Gemeindepräsidenten, 
Gemeinderatsschreiber, Grundbuchbeamte, Denkmalpfleger und Planer für Ausbildungszwecke zu beanspruchen? In den durchgeführten Arbeitswochen hat sich gezeigt, dass Geben und Nehmen durchaus gegenseitig erfolgt. 25 oder mehr Schüler kommen unvoreingenommen und unbelastet und befassen sich intensiv selbst in ihrer Freizeit - mit den Problemen einer Gemeinde. Ihre Fragen decken bei Behörden und Planern Probleme auf, die vorher noch gar nicht erkannt oder noch nicht formuliert wurden. Die Schüler zwingen die mit der täglichen Arbeit bereits überlasteten und nebenamtlich tätigen Behördemitglieder sich mit Fragen der Zukunft ihrer Gemeinde auseinander- sein. zusetzen. Das erleichtert nachher den Planern die Arbeit. Die Umfragen können noch weiter ausgewertet werden. Dann kommen immer wieder Lösungsvorschläge, die realisierbar sind. Oft sind sie verblüfend einfach. Auch sind nicht alle teuer. Von einer unbekannten Schülergruppe können Behördemitglieder leichter etwas annehmen, als von politisch festgelegten Gemeindegliedern. Für viele Behördemitglieder ist der Kontakt mit jungen Menschen, die sich in ihre Probleme hineindenken erfreulich, ja erfrischend, besonders bei gemischten Schulklassen. Ihr Bericht gibt mannigfache Anregung, ohne die Behörde auf etwas zu verpflichten, und all das kann für den weitern Gang einer Planung von bedeutendem Vorteil

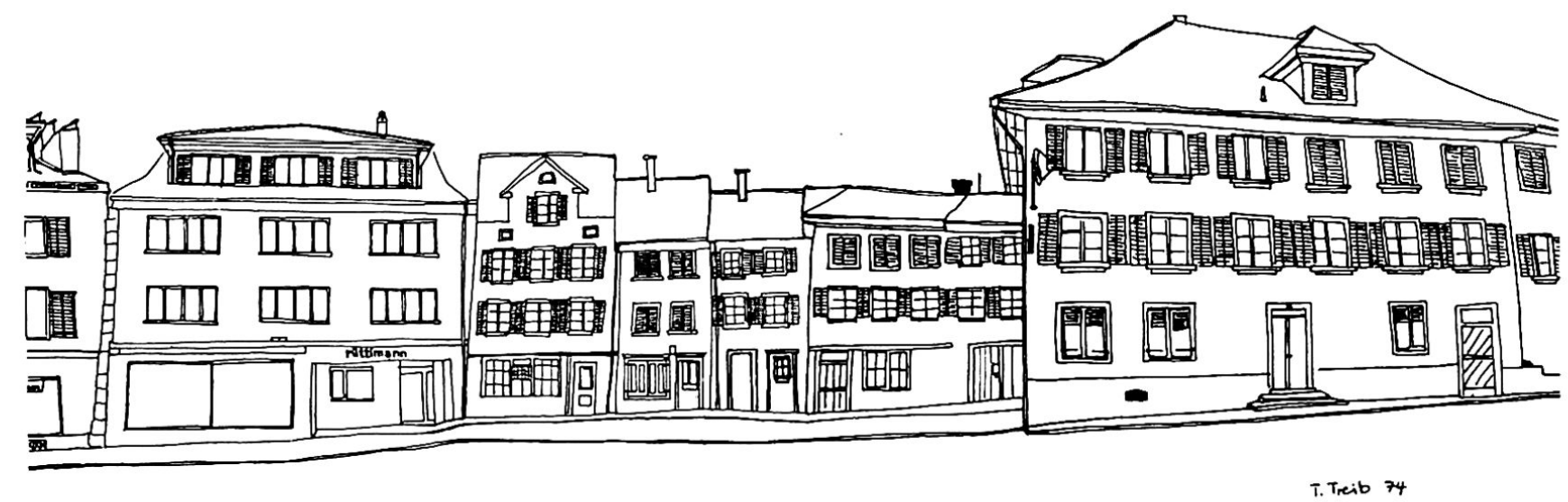

Das nördliche Ende der Hauptstrasse von Sempach bilden vier kleine Bürgerhäuser mit den alten Hof stattmassen und das wohlproportionierte Gebäude der Wirtschaft zum Ochsen. Sie stehen gegenüber der monumentalen Kirche und haben deshalb als Verbindungsglied zu den prächtigen Bürgerhäusern der Hauptstrasse einen ganz besondern Stellenwert. Da die Gebäude schlecht unterhalten sind, denkt die Gemeinde an einen Abbruch. Thomas Treib zeichnete die ganze untere Häuserzeile der Hauptstrasse, um zu zeigen, wie frühere Eingriffe, zum Beispiel der südlich anschliessende Neubau (Rüttimann) störend wirken, und wie durch einen allfälligen Abbruch dieser Gebäude der ganze nördliche Teil der Hauptstrasse und der Platz vor der Kirche ernsthaft gefährdet würden. 